\title{
Encapsulation of granulocyte colony-stimulating factor and granulocyte-macrophage colony-stimulating factor in liposomes prepared by thin film hydration and their transfer to mesenchymal stem cells and cord blood hematopoietic stem cells
}

\author{
Sławomir Lewicki ${ }^{1}$, Monika Leśniak ${ }^{1}$, Agnieszka Sobolewska-Ruta², Aneta Lewicka ${ }^{3}$, \\ Marta Grodzik ${ }^{4}$, Eugeniusz K. Machaj ${ }^{5}$, Marek Saracyn ${ }^{6}$, Jacek Z. Kubiak ${ }^{1,7}$, Zygmunt Pojda ${ }^{8}$
}

\author{
${ }^{1}$ Department of Regenerative Medicine and Cell Biology, Military Institute of Hygiene \\ and Epidemiology, Warsaw, Poland \\ 2Bioengineering Department, The Institute of Biotechnology and Antibiotics, Warsaw, Poland \\ ${ }^{3}$ Laboratory of Food and Nutrition Hygiene, Military Institute of Hygiene and Epidemiology, \\ Warsaw, Poland \\ ${ }^{4}$ Division of Nanobiotechnology, Faculty of Animal Sciences, Warsaw University of Life \\ Sciences, Warsaw, Poland \\ ${ }^{5}$ Polish Stem Cell Bank, Warsaw, Poland \\ ${ }^{6}$ Department of Endocrinology and Isotope Therapy, Military Institute of Medicine, \\ Warsaw, Poland \\ ${ }^{7}$ Univ Rennes, CNRS, IGDR - Institute of Genetics and Development of Rennes, UMR \\ 6290, Cell Cycle Group, Faculty of Medicine, Rennes, France \\ ${ }^{8}$ Department of Regenerative Medicine, Maria Skłodowska-Curie Institute-Oncology \\ Center, Warsaw, Poland
}

Submitted: 15 October 2018; Accepted: 29 June 2019

Online publication: 18 April 2020

Arch Med Sci 2022; 18 (4): 1051-1061

DOI: https://doi.org/10.5114/aoms.2020.94527

Copyright (c) 2020 Termedia \& Banach

\begin{abstract}
Introduction: Cytokines are important immune modulator factors controlling homeostasis of the body and are involved in tissue regeneration after wound healing. The encapsulation of cytokines in liposomes has many advantages potentially useful for their transfer to the cells. Liposomes protect cytokines from neutralization, improving their pharmacokinetics or biologic activity in vivo. They are targeted to specific cell types and may delay the release of cytokines, allowing their sustained paracrine delivery. Their physicochemical characteristics such as size, shape, charge, and stability are important parameters improving bio-distribution and prolonged pharmacokinetics of encapsulated cytokines.

Material and methods: We developed an efficient protocol for the encapsulation of two types of cytokines, granulocyte-macrophage colony-stimulating factor (GM-CSF) and granulocyte colony-stimulating factor (G-CSF), in liposomes that can be stored long term in the active state.

Results: This method allows for the encapsulation of $12-13 \%$ of the total amount of cytokines and $50 \%$ of encapsulated cytokines are entrapped in liposomes of more than $\leq 600 \mathrm{~nm}$ in diameter. We show that in the studied cell lines the liposome-encapsulated cytokines do not affect cell morphology, proliferation or mortality.

Conclusions: The G-CSF or GM-CSF can be delivered to the cells in working concentrations through the encapsulation in the liposomes. Before the clinical application, the efficiency of these liposomes should be confirmed by an in vivo study.
\end{abstract}

Key words: liposomes, granulocyte colony-stimulating factor, granulocytemacrophage colony-stimulating factor, delivery to stem cells, protein transfer to cells in vitro.
Corresponding author: Aneta Lewicka PhD Laboratory of of Food and Nutrition Hygiene Military Institute of Hygiene and Epidemiology

4 Kozielska St

01-163 Warsaw, Poland Phone: +48261853101 E-mail: anet.lewicka@gmail.com 


\section{Introduction}

Cytokines are involved in immune homeostasis and regulation of the inflammatory response. They promote effective control of pathogens, enforce tolerogenic mechanisms and improve tissue regeneration after injury [1]. Granulocyte colonystimulating factor (G-CSF) and granulocyte-macrophage colony-stimulating factor (GM-CSF) are important hematopoietic growth factors with major roles associated with the production and development of granulocytes, monocytes and other antigen-presenting cells [2]. Granulocyte colony-stimulating factor and GM-CSF are also pleiotropic growth factors affecting various functions of immune system cells, e.g. modulation of chemotaxis and migration of neutrophils, phagocytosis of neutrophils and monocytes, surface expression of Fc- and complement-mediated cell-binding (FcyR1, CR-1, CR-3 and adhesion receptor) or modulation of inflammatory reaction [3]. Importantly, G-CSF and GM-CSF participate in the regeneration of injured tissues. It has been shown that the cytokines are involved in the proliferation and migration of endothelial cells and in the development of blood vessels $[4,5]$, the proliferation of keratinocytes [6], fibroblasts and myofibroblasts [7], as well as in collagen synthesis [8].

The wide effects of cytokines on the organism suggest that G-CSF and GM-CSF can be used for the treatment of various diseases. Cytokine therapies are widely used in clinical practice. Cytokine administration increases the number of neutrophils in the peripheral blood and increases the number of peripheral blood stem cells [9]. Granulocyte colony-stimulating factor therapy is successfully used in oncology for the treatment of several advanced neoplasms. Granulocyte colony-stimulating factor or GM-CSF therapies are used for in the following treatments: (1) to treat patients with advanced metastatic disease after chemotherapy to treat or prevent neutropenia, and also in bone marrow transplantation to help to recover from the bone marrow depression induced by chemotherapy [10]; (2) in conjunction with chemotherapy to treat patients with advanced breast carcinoma [11]; (3) to promote the acceleration of myeloid recovery in patients with non-Hodgkin's lymphoma, acute lymphoblastic leukemia, and Hodgkin's disease undergoing autologous stem cell transplantation [2]; (4) to treat brain tumor cells [11]; (5) to treat autoimmune diseases such as inflammatory bowel disease (IBD), Crohn disease and ulcerative colitis [12]; (6) to accelerate myeloid recovery in patients undergoing stem cell transplantation [2]. Granulocyte colony-stimulating factor is also used to decrease the inci- dence of infection in patients with non-myeloid malignancies [2].

However, systematic administration of G-CSF and GM-CSF cytokines can produce significant changes in the bone marrow and peripheral blood WBCs. The effects of G-CSF and GM-CSF on peripheral blood neutrophils include toxic granulation, increased level of granulocytes and their vacuolation, and abnormalities of nuclear segmentation. Other less common features include binucleate promyelocytes and myelocytes, giant myeloid precursors, and rarely, the development of bone marrow fibrosis [9]. Granulocyte-macrophage colony-stimulating factor may also cause monocytosis and eosinophilia. Some trials using very high doses of GM-CSF were often associated with adverse effects such as pericarditis and thrombosis [13]. In order to avoid toxic side-effects associated with systemic cytokine administration, cytokines can be encapsulated within the liposomes and delivered locally. Liposomes are vesicles composed of one or more lipid membranes surrounding an aqueous lumen. Liposomal formulations have been used to encapsulate and deliver various therapeutics, such as small molecules, proteins and oligonucleotides [1]. The use of liposomes improves the biological activity of encapsulated compounds by protecting them from cell metabolism and inactivation in the plasma, increasing their half-life or by improving their tissue-specific biodistribution $[14,15]$.

The main aim of the present work was to characterize and examine the effect of G-CSF and GM-CSF loaded liposomes on mesenchymal stem cells (isolated from the umbilical cord stem cells [UCSC]) and cord blood hematopoietic stem cells (CBHSC). The cells used in this study are well-defined primary stem cells lines, which perform various functions in the body. The UCSC are mesenchymal stem cells, which exhibit the capacity to differentiate into mesodermal (osteocytes, adipocytes, and chondrocytes), ectodermal (neurocytes) and endodermal (hepatocytes) lineages [16]. The UCSC express cell surface markers such as CD29, CD73, CD90, CD105 and do not express CD14, CD34, CD45 and HLA (human leucocyte antigen)-DR [17]. The CBHSC are hematopoietic stem cells, which are able to differentiate into hematopoietic stem cell populations (white or red blood cells and platelets) [18]. The CBHSC express cell surface markers such as CD34, HLA-DR and c-kit tyrosine kinase receptor [19]. Because our previous studies showed that negatively charged liposomes have the lowest cytotoxicity and do not affect hematopoietic cell proliferation [20] we chose this type of liposomes for our present study. 


\section{Material and methods}

\section{Stem cells}

\section{Mesenchymal stromal cell}

Umbilical cord stem cells were isolated as previously described [21]. Briefly, umbilical cord fragments were isolated after childbirth (the protocol was accepted by the local Ethical Committee, permit no. KB 70/2012). Fragments of Wharton jelly were transferred to the culture flask $\left(75 \mathrm{~cm}^{2}\right.$, $\mathrm{BD}$ Falcon, Poland) and maintained in growth medium (DMEM with GlutMax, $20 \%$ of FBS and $50 \mathrm{UI} / \mathrm{ml}$ of antibiotics: penicillin/streptomycin; all from Gibco, Poland). Cells were passaged 3-5 times before use. After the $3^{\text {rd }}$ passage, the cells were phenotyped for the presence of mesenchymal stem cell markers CD29, CD34, CD45, CD73, CD90, CD105, CD106 (all antibodies were purchased from BD, Poland).

All markers were analyzed by flow cytometry (FACS Caliber, BD, USA) as previously described [21]. $95 \%$ of cells exhibited strong expression of CD29 and CD90; high CD73 and CD105; week expression of CD106 and lack of expression of CD34 and CD45 markers, which is consistent with the results described in the literature [17].

Results of flow cytometry analysis are presented in Supplementary Figure S1.

\section{Cord blood hematopoietic stem cells}

Cord blood hematopoietic stem cells were isolated as previously described [11]. Cord blood samples were collected during delivery with the mothers' consent (the protocol was accepted by the local Ethical Committee). The mononuclear cells were isolated by Ficoll-Uropoline sedimentation (Stem Cell Technologies, UK) and frozen in liquid nitrogen $\left(-170^{\circ} \mathrm{C}\right)$ until further use.

\section{Granulocyte colony-stimulating factor and granulocyte-macrophage colony- stimulating factor}

Lyophilized G-CSF and GM-CSF cytokines were purchased from Peprotech IMC (USA). Cytokines were dissolved in phosphate-buffered saline (PBS, Gibco, Poland) with $0.1 \%$ human albumin (Octapharma, Poland) in the concentration of $100 \mathrm{ng} / \mathrm{ml}$, aliquoted and stored at $-80^{\circ} \mathrm{C}$ until use.

\section{Granulocyte colony-stimulating factor} and granulocyte-macrophage colonystimulating factor concentration analysis

Granulocyte colony-stimulating factor or GMCSF concentration in all test was analyzed by an ELISA kit (R\&D Systems, USA) according to the manufacturer's protocol. To obtain the values within a detection range of ELISA kits the G-CSF samples were diluted $1: 4$ and the GM-CSF samples were diluted 1 : 20. Each sample in ELISA analysis was added in duplicate.

\section{Production of granulocyte colony-stimulating factor or granulocyte-macrophage colony- stimulating factor loaded liposomes}

Liposomes were produced by the hand-shaking method [22]. Due to the previously observed strong cytotoxic effect of positive-charge and neutral-charge liposomes on all types of tested cells, only negative-charge liposomes were selected for cytokine encapsulation experiments [11].

According to the previously developed protocol, one dose of cytokine encapsulated in the liposomes was composed of: L- $\alpha$-phosphatidylcholine (100 mg, Sigma Aldrich, Poland, cat. no. P3556), cholesterol (5.6 mg, Sigma Aldrich, Poland, cat. no. C8667) and dihexadecyl phosphate $(8.0 \mathrm{mg}$, Sigma Aldrich, Poland, cat. no. D2631). Reagents were suspended in $15 \mathrm{ml}$ of chloroform (Sigma Aldrich, Poland) in a 250-ml round-bottom flask, which was rotary evaporated (R-300 Rotavapor, Büchi, Poland, 60 RPM) in a water bath at $30^{\circ} \mathrm{C}$ to obtain a thin dry film. The residual solvent was removed by vacuum evaporation for $1 \mathrm{~h}$. The only modification of the previously used method was the addition of $1 \mathrm{ml}$ of G-CSF or GM-CSF stock solution $(100 \mathrm{ng} / \mathrm{ml})$ dissolved in $10 \mathrm{ml}$ of PBS with $0.1 \%$ human albumin. The flask was rotated in an evaporator at $60 \mathrm{rpm}$ for $30 \mathrm{~min}$ at room temperature. All tests on liposomes were performed after overnight incubation $\left(4^{\circ} \mathrm{C}\right)$. To avoid contamination from the cytokines not encapsulated in the liposomes, the cytokine-loaded liposome suspension was centrifuged at $10,000 \mathrm{~g}$ for $1.5 \mathrm{~h}$, the supernatant was removed and the pellet was resuspended in PBS. This procedure was repeated four times.

Supernatants from the last centrifugation were collected, and the absence of free G-CSF or GMCSF was confirmed by ELISA analysis (data not shown).

\section{Granulocyte colony-stimulating factor and granulocyte-macrophage colony- stimulating factor content in the liposomes}

\section{Efficiency of cytokine encapsulation}

Cytokine concentration was measured with ELISA tests (R\&D system, USA). To evaluate the efficiency of G-CSF or GM-CSF encapsulation in the liposomes we assessed cytokine concentration in (1) fresh defrosted cytokine solution (control), (2) liposome-containing PBS/albumin "opened" or not by treatment (20 $\mathrm{min}$ at room temperature) with 5\% Tween 20, (3) liposomes after centrif- 
ugation "opened" by treatment $(20 \mathrm{~min}$ at room temperature) with 5\% Tween 20, (4) supernatant after liposome centrifugation. To assess the possible effect of Tween 20 (Sigma Aldrich, Poland) the Tween was also added to the control samples. The results are presented as mean \pm standard deviation (SD). All samples were analyzed in sextuplets.

In order to check if we can produce the nanoliposomes with encapsulated cytokines, the liposomecontaining PBS/albumin solution was homogenized (15 $\mathrm{ml}$ of the solution, per $5 \mathrm{~min}$ ) in EmulsiFlex C3 machine (Avestin, Australia). Then cytokine concentrations of homogenized liposomes were analyzed in sextuplets.

\section{Efficiency of cytokine encapsulation in liposomes of various sizes}

The minimum diameter of liposomes encapsulating chemical particles depends on many parameters and cannot be predicted theoretically [23]. Therefore G-CSF or GM-CSF prepared liposomes were filtered through polycarbonate filters (Millipore, Poland), with pore size $200 \mathrm{~nm}, 400 \mathrm{~nm}$, and $600 \mathrm{~nm}$. The concentration of cytokines encapsulated in $\leq 200 \mathrm{~nm}, \leq 400 \mathrm{~nm}$, and $\leq 600 \mathrm{~nm}$ diameter liposomes was analyzed using an ELISA kit. Results are presented as the \% of the whole liposome population \pm SD. All samples were analyzed in sextuplets.

Biological activity of liposome encapsulated cytokines (granulocyte colony-stimulating factor, granulocyte-macrophage colonystimulating factor)

\section{Cytotoxic effect of liposomes on umbilical cord stem cells growth}

The cytotoxic effect of cytokine-loaded liposomes on UCSC growth was evaluated by proliferation assay. UCSC cells were seeded in 24-well culture plates (BD Falcon, Poland) in a concentration of $2 \times 10^{4}$ cells per well in DMEM with GlutaMAX, $20 \%$ of FBS with $50 \mathrm{UI} / \mathrm{ml}$ of penicillin/streptomycin. Following $24 \mathrm{~h}$ of incubation, the liposome suspension (liposomes in PBS supplemented with $0.1 \%$ of albumin) was added. Liposome suspension accounted for $5 \%$ of the total volume of culture medium, i.e. about $0.38 \mu \mathrm{g}$ of liposomes/ $\mu$ l. After 5 days of culture, the proliferation assay was performed. We used 4 independent tests: cell number count (Bürker chamber), MTT assay, according to Rokicki et al. [24], NR-assay and SRB assay, according to Lewicki et al. [20]. Each assay was performed in triplicate $(n=6)$. The results are presented as the percentage of control values (mean $\pm \mathrm{SD})$.

\section{Effect of liposome encapsulated granulocyte colony-stimulating factor and granulocyte-macrophage colony- stimulating factor on cord blood hematopoietic stem cells growth}

Granulocyte colony-stimulating factor or GMCSF-loaded liposomes were used as cytokine source in in vitro clonal cultures of hematopoietic cells (CBHSC). Cord blood hematopoietic stem cells cells were cultured in 24 -well plates $\left(1 \times 10^{4}\right.$ cells per well) in commercial methylcellulose medium. Two different methylcellulose media were used (all from Stem Cell Technologies, UK): H4230 without growth factors and without Epo, and H4330 without growth factors and with Epo. $50 \mu$ l of liposome suspension (loaded with G-CSF or GM-CSF) were added to $950 \mu \mathrm{l}$ of methylcellulose medium and mixed thoroughly prior to cell addition. Before liposome addition, the concentration of encapsulated cytokines was evaluated. For each study, approximately $10 \mathrm{ng} / \mathrm{ml}$ of G-CSF or GM-CSF added to $50 \mu \mathrm{l}$ of liposome solution was used. The positive control consisted of $50 \mu \mathrm{l}$ of pure cytokines in PBS with $0.1 \%$ human albumin (at concentration $10 \mathrm{ng} / \mathrm{ml}$ ). Controls consisted of $50 \mu \mathrm{l}$ of PBS with $0.1 \%$ human albumin (without cytokines, C1) or $50 \mu \mathrm{l}$ of "empty" liposome suspension. The number of cells (formed by mixed cells Mix-CFCs, erythroid cells - BFU-Es or granulocyte-macrophage cells GM-CFCs) was analyzed after 14 days of culture. Results were presented as a percentage of control cells' (C1) values at 14 days of culture. All experiments were performed in triplicate $(n=6)$. The results are presented as the percentage of control values (mean $\pm \mathrm{SD}$ ).

\section{Storage effect}

We evaluated the effect of storage temperature of G-CSF- or GM-CSF-loaded liposomes on the activity of cytokines extracted from liposomes. We used three common storage temperatures: $-20^{\circ} \mathrm{C},+4^{\circ} \mathrm{C}$ and $+24^{\circ} \mathrm{C}$. Cytokine content was determined at the beginning of the experiment, and subsequently at the $1^{\text {st }}, 7^{\text {th }}, 14^{\text {th }}$, and $28^{\text {th }}$ day of storage. The cytotoxic effect of the prepared liposomes (cell number, MTT, NR, and SRB assay) was assessed as described above. The results are presented as the mean \pm standard deviation. All samples were analyzed in sextuplets.

\section{Statistical analysis}

Statistical evaluation of the results was performed using the $t$-test and one-way ANOVA with Bonferroni correction (in the case of a normal distribution) or non-parametric Kruskal-Wallis and Mann-Whitney $U$ tests (in the case of an abnormal distribution). Assessment of the distribution of the 
Encapsulation of granulocyte colony-stimulating factor and granulocyte-macrophage colony-stimulating factor in liposomes prepared by thin film hydration and their transfer to mesenchymal stem cells and cord blood hematopoietic stem cells

Table I. Granulocyte colony-stimulating factor (G-CSF) or granulocyte-macrophage colony-stimulating factor

(GM-CSF) concentration in phosphate-buffered saline presented as concentration in $\mathrm{pg} / \mathrm{ml}$ and \% of the control value (fresh defrosted G-CSF or GM-CSF solution)

\begin{tabular}{|c|c|c|c|}
\hline Factor & Mean $[\mathrm{pg} / \mathrm{ml}]$ & SD & $\%$ of control \\
\hline G-CSF solution in PBS & 10526 & 368 & 100.0 \\
\hline G-CSF solution in PBS + Tween & 10896 & 467 & 103.5 \\
\hline G-CSF liposomes in PBS + Tween & 9274 & 619 & 88.1 \\
\hline G-CSF liposomes in PBS & 7904 & 237 & 75.1 \\
\hline PBS after centrifugation & 5604 & 394 & 53.2 \\
\hline Centrifugation-isolated liposomes & 1292 & 571 & 12.3 \\
\hline Homogenized liposomes & 1314 & 388 & 12.5 \\
\hline Homogenized liposomes + Tween & 1624 & 184 & 15.0 \\
\hline GM-CSF solution in PBS & 10324 & 468 & 100.0 \\
\hline GM-CSF solution in PBS + Tween & 10376 & 64 & 100.5 \\
\hline GM-CSF liposomes in PBS + Tween & 8474 & 1750 & 82.1 \\
\hline GM-CSF liposomes in PBS & 7058 & 1042 & 68.4 \\
\hline PBS after centrifugation & 7132 & 988 & 69.1 \\
\hline Centrifugation-isolated liposomes & 1386 & 580 & 13.4 \\
\hline Homogenized liposomes & 7102 & 266 & 68.8 \\
\hline Homogenized liposomes + Tween & 7330 & 158 & 71.0 \\
\hline
\end{tabular}

G-CSF - granulocyte colony-stimulating factor, PBS - phosphate-buffered saline, GM-CSF - granulocyte-macrophage colony-stimulating factor.

Table II. Mean percentage \pm SD of cytokines encapsulated in different size liposomes

\begin{tabular}{|c|c|c|c|c|}
\hline \multirow[t]{3}{*}{ Growth factor } & \multicolumn{4}{|c|}{ Liposomes diameter } \\
\hline & $\leq 200 \mathrm{~nm}$ & $200-400 \mathrm{~nm}$ & $\leq 600 \mathrm{~nm}$ & Whole liposome population \\
\hline & \multicolumn{4}{|c|}{$\%$ of encapsulated cytokines } \\
\hline GM-CSF & $15.3 \pm 1.8$ & $32.0 \pm 8.6$ & $47.4 \pm 20.1$ & $100 \%$ \\
\hline G-CSF & $17.6 \pm 2.2$ & $28.3 \pm 6.7$ & $42.9 \pm 10.5$ & $100 \%$ \\
\hline
\end{tabular}

GM-CSF - granulocyte-macrophage colony-stimulating, G-CSF - granulocyte colony-stimulating factor.

data was evaluated using the Shapiro-Wilk test. GraphPad Prism software was used to carry out these tests (version 7; GraphPad Software, Inc., La Jolla, CA, USA). $P<0.05$ was considered a statistically significant difference.

\section{Results}

\section{Efficiency of cytokine encapsulation}

The analysis of the efficiency of encapsulation of G-CSF and GM-CSF is presented in Table I. The deduction of the value of cytokine concentration in PBS from the value of the concentration after cytokine release to PBS by opening liposomes with Tween 20 represented the amount of cytokine encapsulated in the liposomes. Results of the anal- yses showed that $12-13 \%$ of the total amount of cytokines was successfully encapsulated. We are aware that this is not very high encapsulation efficiency in comparison to the efficiencies obtained in other studies $[25,26]$; however, our protocol is relatively simple and does not contain the postencapsulation steps, such as the freeze-thawing cycles, which increase cytokine concentration in liposomes. Homogenized liposomes contained less than $3 \%$ of encapsulated cytokines, and therefore were not used in further analysis.

Effect of liposome size on the concentration of encapsulated cytokines

Nearly $50 \%$ of cytokines (GM-CSF $-47.4 \%$ and G-CSF - 42.9\%) were encapsulated in $\leq 600 \mathrm{~nm}$ 
liposomes, while only $32 \%$ of GM-CSF and $28.3 \%$ of G-CSF were encapsulated in 200-400 nm liposomes. The $\leq 200 \mathrm{~nm}$ liposomes contained less than $20 \%$ of total amounts of cytokines (G-CSF - $17.6 \%$ and GM-CSF - 15.3\%). The analysis of the percentage of cytokines encapsulated in different size liposomes is summarized in Table II.

\section{Biological activity of cytokines (granulocyte colony-stimulating factor, granulocyte- macrophage colony-stimulating factor) encapsulated in liposomes}

\section{Effect of liposomes on umbilical cord stem cells growth}

The addition of freshly prepared G-CSF or GMCSF-loaded liposomes into the cultures of UCSC cells did not result in any change in cell morphology. We also did not observe any change in the proliferation and cell death in comparison to the control cells (Figure 1).

\section{Effect of liposomes on cord blood hematopoietic stem cells growth}

\section{Methylcellulose medium without erythropoietin}

Cord blood hematopoietic stem cells cultured in methylcellulose medium without Epo and without growth factors (H4230, Stem Cell Technologies) differentiated only into granulocyte-macrophage cell colonies (GM-CFU). Co-culture of CBHSC cells with G-CSF-loaded liposomes or soluble G-CSF significantly increased the number of cells after 14 days of incubation in $\mathrm{H} 4230$ methylcellulose medium in comparison to the control conditions (cells without cytokine treatment) by $21 \%-p<0.01$ or $23 \%$, respectively $p<0.001$ ). Also, the soluble fraction of GM-CSF-loaded liposomes and pure GM-CSF increased the number of cells $(126 \%$ and $166 \%$ respectively, $p<0.001)$. The increased number of colonies after treatment

A

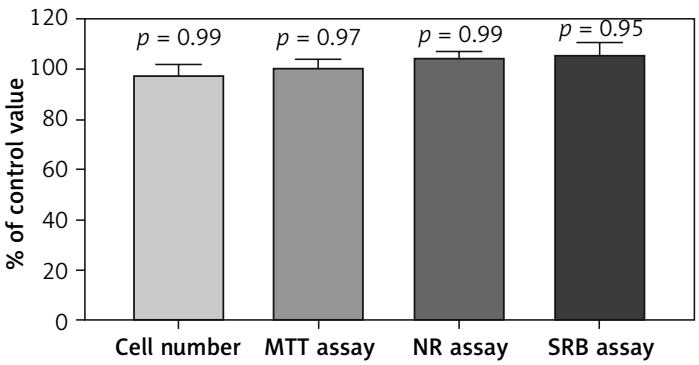

with GM-CSF-loaded liposomes was however lower than after treatment with soluble GM-CSF and the difference was statistically significant $(p=0.032)$. The empty liposomes strongly reduced cell proliferation in control conditions $(30 \%$, $p<0.001$ ) (Figure 2 A).

\section{Methylcellulose medium with erythropoietin}

Cord blood hematopoietic stem cells cultured in methylcellulose medium containing Epo without growth factors ( 14330$)$ differentiated into three types of colonies: mixed cells (Mix-CFU), erythroid cells (BFU-E), or granulocyte-macrophage cells (GMCFU). Co-culture of CBHSC cells with cytokine-loaded liposomes significantly $(p>0.001)$ increased the number of Mix-CFU and GM-CFU colonies after 14 days of incubation. The liposome loaded with cytokines did not affect the growth of BFU-E cells in comparison to the control (Figure $2 \mathrm{~B}$ ).

\section{Storage effect \\ Concentration of granulocyte colony- stimulating factor or granulocyte- macrophage colony-stimulating factor}

Although it is known that storage below $-70^{\circ} \mathrm{C}$ or lyophilization guarantees the unlimited lifetime of proteins [27], the typical storage conditions for the drugs and wound dressings are $-20^{\circ} \mathrm{C}$ (freezer), $+4^{\circ} \mathrm{C}$ (fridge) or $+24^{\circ} \mathrm{C}$ (room temperature). Using the ELISA test, we analyzed the effect of different storage temperatures on the concentration of the active form of G-CSF and GM-CSF encapsulated in the liposomes, which were suspended in PBS with albumin. The measurements were performed at weekly intervals within 28 days of storage.

In the control condition (PBS with G-CSF) the storage at $24^{\circ} \mathrm{C}$ caused a fast decrease of concentration of G-CSF (at the $28^{\text {th }}$ day only about $45 \%$ of cytokine was found by ELISA). At $4{ }^{\circ} \mathrm{C}, 49 \%$ and at $-20^{\circ} \mathrm{C}-75 \%$ of cytokine was found at the $28^{\text {th }}$ day of the experiment. Encapsulation of GM-

B

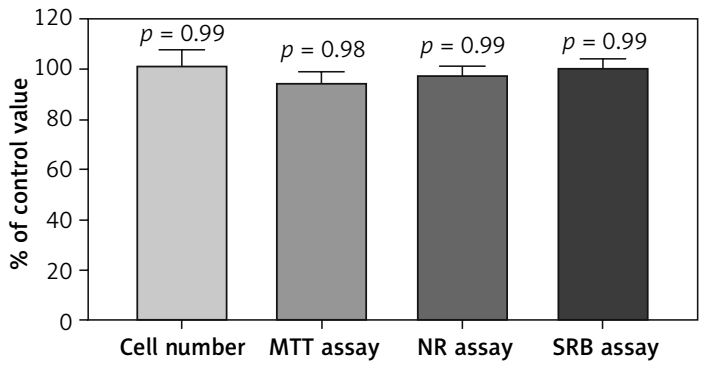

Figure 1. Effect of A - granulocyte colony-stimulating factor (G-CSF) or B - granulocyte-macrophage colony-stimulating factor (GM-CSF) loaded liposomes on proliferation of umbilical cord stem cells cells as assessed by cell number, MTT, NR and SRB assays. Assays were performed in triplicate $(n=6)$. Results are shown as a mean percentage \pm SD of control values. $P$ - probability value, statistical significance $p<0.05$ 
A

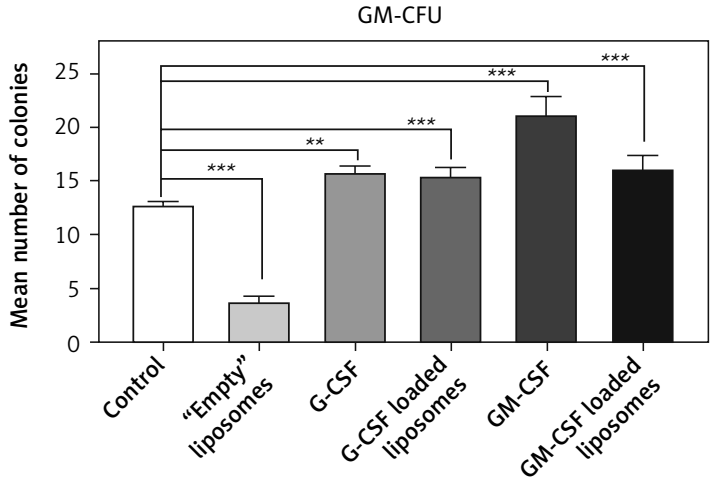

B
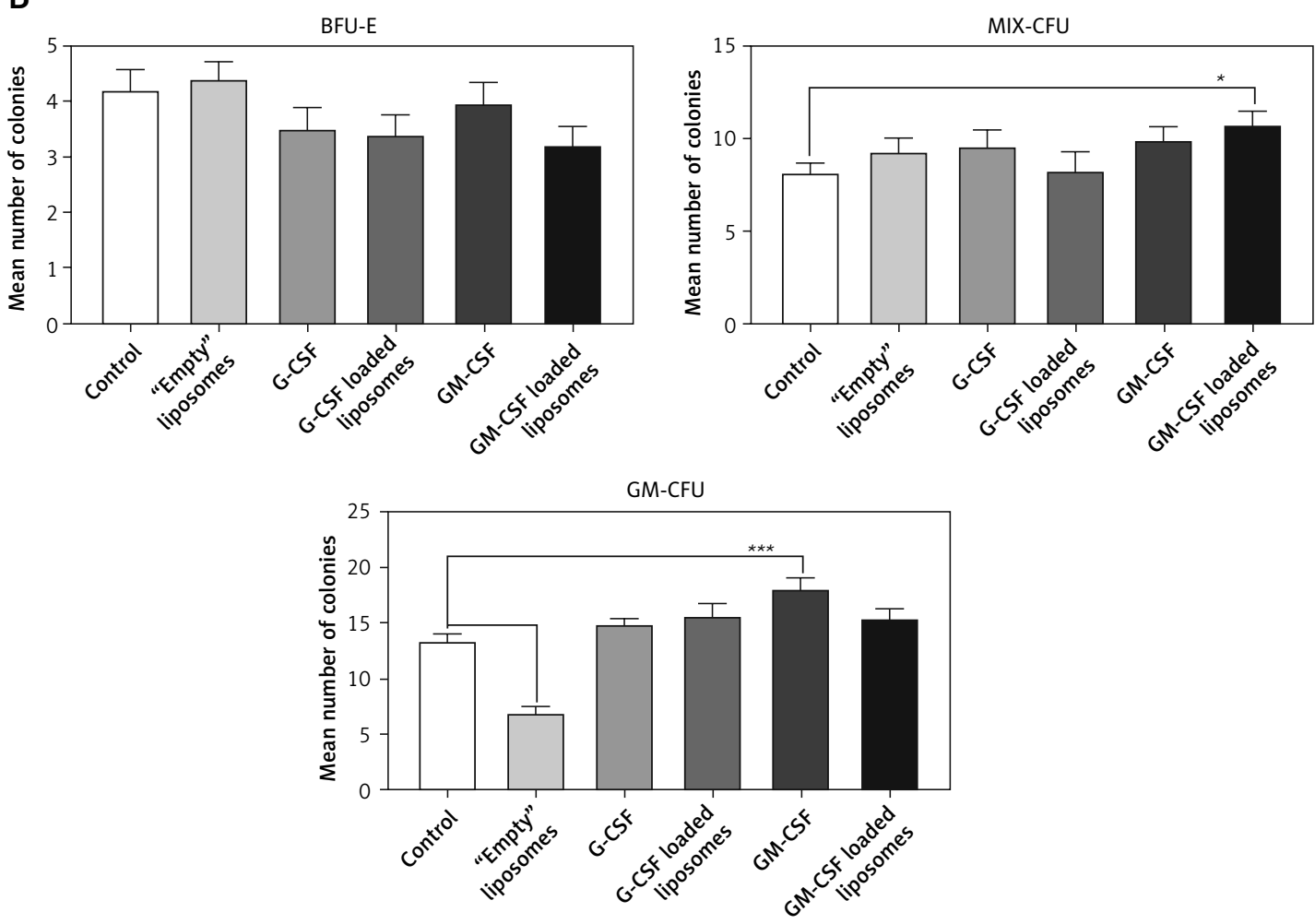

Figure 2. The effect of liposome-encapsulated granulocyte-macrophage colony-stimulating factor (GM-CSF) and granulocyte colony-stimulating factor (G-CSF) cytokines on hematopoietic stem cell (CBHSC) proliferation. Liposomes (at final concentration 5\%) were added to the $\mathrm{H} 4230$ methylcellulose medium without Epo (Stem Cell Technologies) (A) or to the $\mathrm{H} 4330$ methylcellulose medium without growth factors supplemented with Epo (B). Results are presented as number of granulocyte/macrophages (GM) (A) or BFU-E, Mix-CFU and GM-CU colonies (B). Results are shown as a mean \pm SEM. Assays were performed in triplicate $(n=6) .{ }^{*} p<0.05 ;{ }^{* *} p<0.01 ;{ }^{* * *} p<0.001$

CSF in liposomes resulted in a slower reduction of G-CSF during $4^{\circ} \mathrm{C}$ and $24^{\circ} \mathrm{C}$ storage $(59 \%$ and $54 \%$ respectively). Interestingly, freezing at $-20^{\circ} \mathrm{C}$ resulted in the preservation of approximately $90 \%$ of G-CSF at day 28 , which was about $15 \%$ higher than in the control. A summary of these results is presented in Figure $3 \mathrm{~A}$.

In contrast to the relatively low G-CSF stability, the GM-CSF was much more stable at all tested temperatures (Figure $3 \mathrm{~B}$ ). At $24^{\circ} \mathrm{C}$ the concentration of cytokine was about $70 \%$, at $4^{\circ} \mathrm{C}$ about $79 \%$ and at $-20^{\circ} \mathrm{C}$ about $91 \%$ of fresh cytokine concentration. Liposome encapsulation of GM-CSF caused increased stability of GM-CSF concentration as compared to the fresh cytokine at the $28^{\text {th }}$ day of the experiment.

\section{Cytotoxic effect on umbilical cord stem cells growth}

The addition of G-CSF- or GM-CSF-loaded liposomes to the UCSC cells did not affect cell morphology, number or mortality (Figures $4 \mathrm{~A}, \mathrm{~B}$ ). We did not observe any effect on UCSC proliferation when the soluble or liposome encapsulated G- or GM-CSF cytokines were used (data not shown). 
A
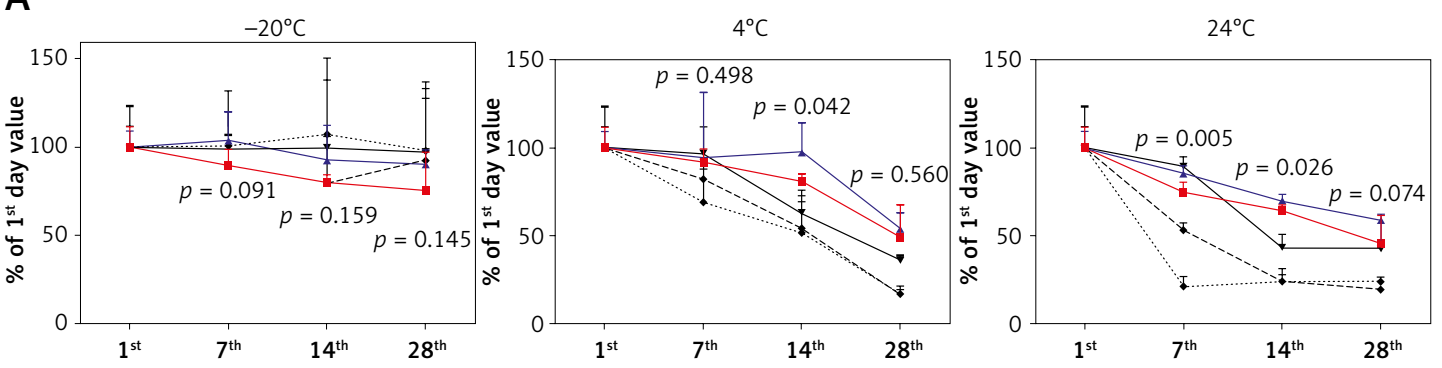

B
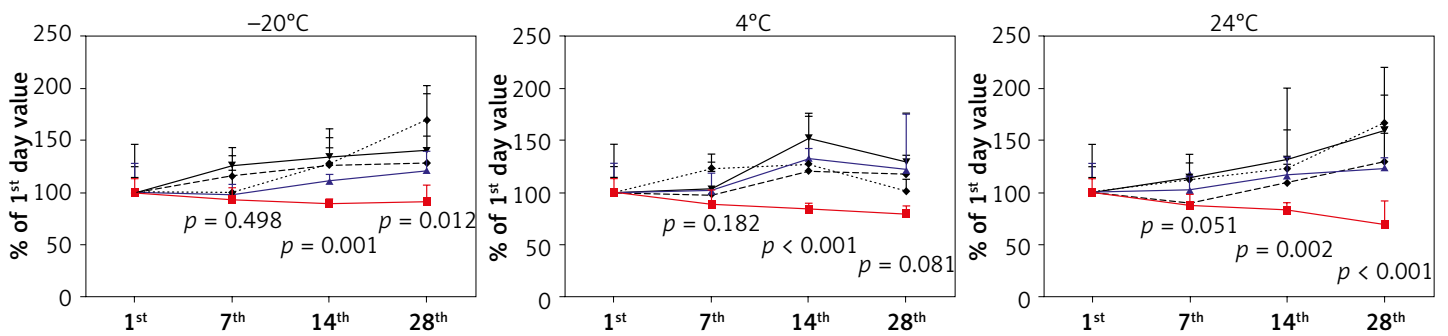

$\rightarrow$ - Control solution $\rightarrow$ Liposomes (all)

$\rightarrow<600 \mathrm{~nm}-\bullet-200-400 \mathrm{~nm}$

$\cdot \cdot 200 \mathrm{~nm}$

Figure 3. The effect of temperature and time of storage on granulocyte colony-stimulating factor (G-CSF) (A) or granulocyte-macrophage colony-stimulating factor (GM-CSF) (B) loaded liposomes. The concentration of cytokines extracted from liposomes was analyzed by ELISA test. Cytokine concentration was tested at the beginning of the experiment, and subsequently at the $1^{\text {st }}, 7^{\text {th }}, 14^{\text {th }}$, and $28^{\text {th }}$ day of storage. Comparison between control solution and cytokine-loaded liposomes. $P$ - probability value, statistical significance $p<0.05$

\section{Discussion}

Liposomes are a well-known method of cytokine delivery to the cells used in treatments of many infectious diseases [28]. Liposomes offer several advantages over conventional delivery systems, especially for the parenteral administration (i.e. intravenous and intraperitoneal injection) [29]. They are targeted to the specific cell types and the delayed release of cytokine from the liposomes results in sustained paracrine-like delivery of the cytokines. The most important role of liposomes, however, is to protect the cytokines or other encapsulated molecules from the biodegradation [29]. The encapsulation in liposomes results in different biodistribution, and improves the pharmacokinetics, biological activity in vivo, and the therapeutic efficiency in comparison to the non-encapsulated drugs [23]. The limitation of liposome methodology is a potential reduction of bioavailability of the drug, saturation of the immune competent cells with lipids, and potentially increased toxicity of some drugs due to their enhanced interactions with particular cells. The benefits and limitations of liposome drug carriers critically depend on the type of interactions of liposomes with cells and their fate in the body [30].

Anderson et al. [31] showed that the liposome composition is important and can be tailored to deliver bioactive cytokines such as IL-1 $\alpha$, IL-2, IL-6,
IFN- $\gamma$ or GM-CSF to their target locations. In order to address the limitations and therapeutic potential of encapsulated cytokines, the cytokine release rate, the time of residence at the injection site, the biodistribution and bioactivity have to be understood [1]. Here we have developed an efficient protocol for the liposome encapsulation of GM-CSF and G-CSF cytokines. The encapsulated cytokines are stable and active for a long period of time. The GM-CSF cytokine is clinically approved for the acceleration of myeloid recovery in patients with non-Hodgkin's lymphoma, acute lymphoblastic leukemia, and Hodgkin's disease undergoing autologous stem cell transplantation. GM-CSF is used for neutropenia associated with stem cell transplantation. The G-CSF cytokine is used for the treatment of neutropenia and for the mobilization of peripheral hematopoietic progenitor cells for stem cell transplantation [2].

The physicochemical parameters of liposomes, such as size, shape, and charge, affect bio-distribution, pharmacokinetics, and life span of encapsulated cytokines [32]. Kedar et al. [25] suggested that liposome-encapsulated GM-CSF and TNF- $\alpha$ might be more efficient immunomodulators than the soluble cytokines. Our protocol allows for encapsulation of $12-13 \%$ of fresh cytokines in the liposomes. In other studies, the encapsulation level of proteins was shown to be between $10 \%$ and $95 \%$ and depended on several factors such as the interaction between the protein and phos- 
A
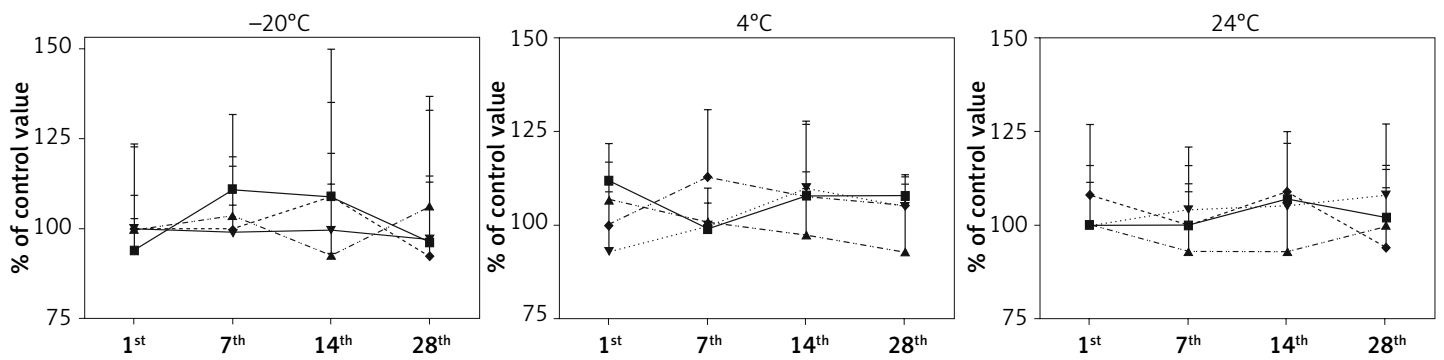

B
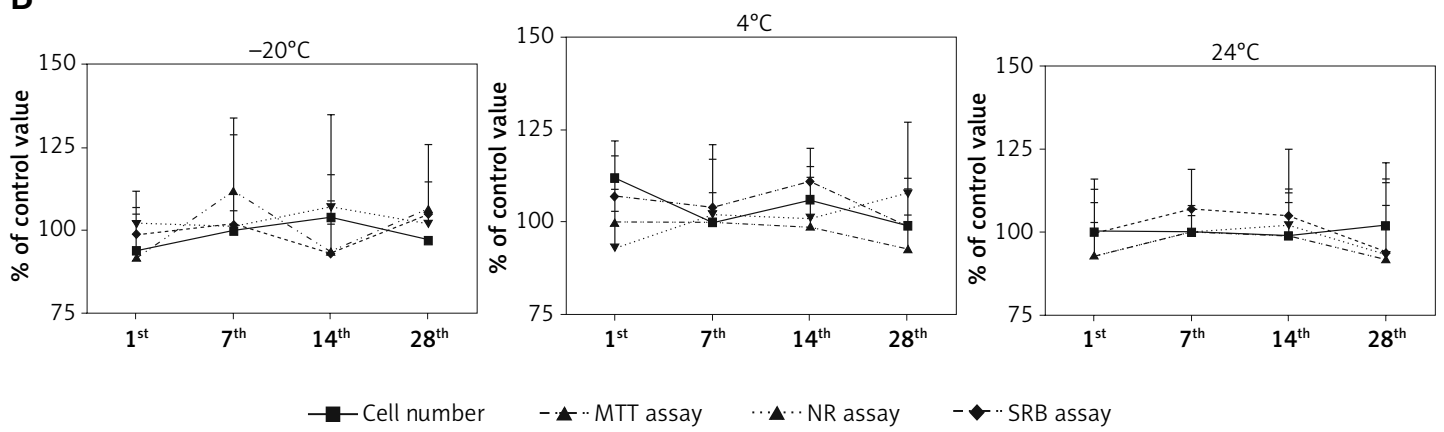

Figure 4. The effect of storage temperature of granulocyte colony-stimulating factor (G-CSF) (A) or granulocytemacrophage colony-stimulating factor (GM-CSF) (B) loaded liposomes on proliferation measured by cell count, MTT, NR and SRB assay. Liposomes were tested at the $1^{\text {st }}, 7^{\text {th }}, 14^{\text {th }}$, and $28^{\text {th }}$ days of storage in differ temperatures

pholipid bilayer, protein mass or electric charge of liposomes [33, 34]. Although $12-13 \%$ is not very high encapsulation efficiency, we obtained liposomes which are nontoxic to the cells and contain biologically active cytokines, efficiently taken up by the cells, which was confirmed by CBHSC assay. The encapsulation efficiency can be easily increased for example by using several freeze-thawing cycles [35]. The positive effect of freeze-thawing may explain a slight increase of GM-CSF concentration, which we observed in the liposomes stored at $-20^{\circ} \mathrm{C}$. We showed that over $50 \%$ of encapsulated cytokines is entrapped in $\leq 600 \mathrm{~nm}$ liposomes. A similar result, about $50-60 \%$ of encapsulation efficiency for G-CSF $\leq 600 \mathrm{~nm}$ liposomes, was obtained by Kiafar et al. [26] for DDPC/cholesterol liposomes prepared by thin layer film hydration.

The major limitation of the liposome delivery system is the instability of the load. For this reason, we examined the effect of storage conditions on the concentration of liposome-encapsulated cytokines. We observed that storage at $24^{\circ} \mathrm{C}$ caused a fast decrease of G-CSF concentration in the liposomes, and freeze-storage at $-20^{\circ} \mathrm{C}$ resulted in the preservation of $90 \%$ of G-CSF concentration for 28 days. It has been shown that the reduced temperature storage from $20^{\circ} \mathrm{C}$ to $4^{\circ} \mathrm{C}$ not only improves the stability of liposomes [36] but also prolongs the viability of biologically active compounds [37]. In contrast to the relatively low G-CSF stability, the GM-CSF was fully stable for
28 days when stored at all temperatures tested. Biological activity of G-CSF/GM-CSF encapsulated in the liposomes was tested on UCSC and CBHSC cells. We did not observe any change in the morphology or cell number in the samples treated with cytokines-loaded liposomes (G-CSF or GMCSF) for the period of at least 1 month, after -20 , +4 and $24^{\circ} \mathrm{C}$ storage.

The most important question of the study was whether the cytokines encapsulated in liposomes remain biologically active and are able to stimulate specific processes in cells after being released from the liposomes. To test this we used umbilical cord hematopoietic stem cells (CBHSC), which are known to respond to G-CSF or GM-CSF supplementation by increasing the number of colonies in the methylcellulose culture. The co-culture of CBHSC cells with G-CSF or GM-CSF-loaded liposomes significantly increased the number of cells after 14 days of incubation in $\mathrm{H} 4230$ methylcellulose medium in comparison to the control conditions $(21 \%-p<0.01$ for G-CSF-loaded liposomes and $26 \% p<0.001$ for GM-CSF loaded liposomes). A similar result (increased number of colonies) was obtained for fresh cytokines: $23 \%, p<0.001$ and $66 \%, p<0.001$ respectively for G-CSF and GM-CSF. Significant differences in the number of GM-CFU colonies (about 30\%, $p=0.032$ ) between the soluble cytokine- and liposome-loaded GM-CSF samples may be the result of increased affinity of the cytokine to the liposome structure. This may also in part explain the higher 
level of the GM-CSF found in the liposomes after the $14^{\text {th }}$ and $28^{\text {th }}$ day of storage. Interestingly, the empty liposomes strongly reduced cell colony number, about $70 \%(p<0.001)$ in comparison to the control (Figure $2 \mathrm{~A})$. The results concerning empty liposomes agree with our previous study, in which addition of liposomes to methylcellulose medium limited in growth factors (H4230) caused a significant reduction of the number of GM-CFU colonies [11]. In contrast, the addition of empty liposomes to methylcellulose medium supplemented with growth factors did not affect GM-CFU colonies. In this study, we also observed this relationship. Empty liposomes caused a reduction of GM-CFU also in other methylcellulose medium used in the study (H4330 methylcellulose medium -limited in growth factors, but with the addition of erythropoietin). However, the addition of cytokines to liposomes eliminated the adverse effects of empty liposomes. The number of colonies in liposome-loaded cytokines was almost similar to that observed after fresh cytokine addition. We did not observe effects of the liposome-loaded cytokines on the number of BFU-E and Mix-CFU colonies.

Taking the evidence together, both cytokines studied here (G-CSF and GM-CSF) encapsulated in liposomes were biologically active and did not cause side effects in the stem cells after prolonged storage. This indicated that we developed an efficient method for cytokine encapsulation. However, before this technology can be applied therapeutically the efficiency of cytokines encapsulation has to be improved.

In conclusion, the presented method allows efficient encapsulation of G-CSF and GM-CSF in liposomes. In all tested temperatures of storage, the cytokines (especially GM-CSF) were more stable when encapsulated in liposomes than in the control solution. The cytokines were released from liposomes and caused a biological effect in the CBHSC culture. These results are very promising for the future use of G-CSF and GM-CSF loaded liposomes in preclinical trials.

\section{Acknowledgments}

The study was supported by the USA Defense Advanced Research Project Agency project no. N1OPC20038 and Polish Ministry of National Defense project “Kościuszko" no: 571/2016/DA.

\section{Conflict of interest}

The authors declare no conflict of interest.

\section{References}

1. Christian DA, Hunter CA. Particle-mediated delivery of cytokines for immunotherapy. Immunotherapy 2012; 4: 425-41.
2. Mehta HM, Malandra M, Corey SJ. G-CSF and GM-CSF in neutropenia. J Immunol 2015; 195: 1341-9.

3. Martins A, Han J, Kim SO. The multifaceted effects of granulocyte colony-stimulating factor in immunomodulation and potential roles in intestinal immune homeostasis. IUBMB Life 2010; 62: 611-7.

4. Zhao J, Chen L, Shu B, et al. Granulocyte/macrophage colony-stimulating factor influences angiogenesis by regulating the coordinated expression of VEGF and the Ang/Tie system. PLoS One 2014; 9: e92691.

5. Natori T, Sata M, Washida M, Hirata Y, Nagai R, Makuuchi M. G-CSF stimulates angiogenesis and promotes tumor growth: potential contribution of bone marrowderived endothelial progenitor cells. Biochem Biophys Res Commun 2002; 297: 1058-61.

6. Mueller MM, Fusenig NE. Constitutive expression of G-CSF and GM-CSF in human skin carcinoma cells with functional consequence for tumor progression. Int J Cancer 1999; 83: 780-9.

7. Carr MJ, Li Y, Rezakhanlou AM, Ghahary A. Keratinocyte-releasable factors stimulate the expression of granulocyte colony-stimulating factor in human dermal fibroblasts. J Cell Biochem 2017; 118: 308-17.

8. Lim JY, Choi BH, Lee S, Jang YH, Choi JS, Kim YM. Regulation of wound healing by granulocyte-macrophage colony-stimulating factor after vocal fold injury. PLoS One 2013; 8: e54256.

9. O'Malley DP, Hsi ED, Smith L, Fedoriw Y. 3-non-neoplastic morphologic abnormalities of white blood cells and macrophages. Hematopathology (Third Edition). A Volume in the Series: Foundations in Diagnostic Pathology Foundations in Diagnostic Pathology 2018; 106-117.e2.

10. Battiwalla M, McCarthy PL. Filgrastim support in allogeneic HSCT for myeloid malignancies: a review of the role of G-CSF and the implications for current practice. Bone Marrow Transplant 2009; 43: 351-6.

11. Bottoni U, Trapasso F. The role of G-CSF in the treatment of advanced tumors. Cancer Biol Ther 2009; 8: 1744-6.

12. Dieckgraefe BK, Korzenik JR. Treatment of active Crohn's disease with recombinant human granulocyte-macrophage colony-stimulating factor. Lancet 2002; 360: 1478-80.

13. Stern AC, Jones TC. The side-effect profile of GM-CSF. Infection 1992; 20 Suppl 2: S124-7.

14. Jämbeck JP, Eriksson ES, Laaksonen A, Lyubartsev AP, Eriksson LA. Molecular dynamics studies of liposomes as carriers for photosensitizing drugs: development, validation, and simulations with a coarse-grained model. J Chem Theory Comput 2014; 10: 5-13.

15. Luo LH, Zheng PJ, Nie H, et al. Pharmacokinetics and tissue distribution of docetaxel liposome mediated by a novel galactosylated cholesterol derivatives synthesized by lipase-catalyzed esterification in non-aqueous phase. Drug Deliv 2016; 23: 1282-90.

16. Ullah I, Subbarao RB, Rho GJ. Human mesenchymal stem cells - current trends and future prospective. Biosci Rep 2015; 35: pii: e00191.

17. Maleki M, Ghanbarvand F, Reza Behvarz M, Ejtemaei M, Ghadirkhomi E. Comparison of mesenchymal stem cell markers in multiple human adult stem cells. Int J Stem Cells 2014; 7: 118-26.

18. Ng AP, Alexander WS. Haematopoietic stem cells: past, present and future. Cell Death Discov 2017; 3: 17002.

19. Hordyjewska A, Popiołek $Ł$, Horecka A. Characteristics of hematopoietic stem cells of umbilical cord blood. Cytotechnology 2015; 67: 387-96.

20. Lewicki S, Leśniak M, Machaj E, et al. Physical properties and biological interactions of liposomes developed 
as a drug carrier in the field of regenerative medicine. J Liposome Res 2017; 27: 90-8.

21. Leśniak M, Zdanowski R, Kubiak J, et al. Effects of hexachlorophene, a chemical accumulating in adipose tissue, on mouse and human Mesenchymal Stem Cells. Tissue Eng Regen Med 2018; 15: 211-22.

22. Dwivedi C, Yadav R, Tiwari SP. Satapathy T, Ro A. Role of liposome in novel drug delivery system. J Drug Del Thera (JDDT) 2014; 4: 116-29.

23. Bozzuto G, Molinari A. Liposomes as nanomedical devices. Int J Nanomedicine 2015; 10: 975-99.

24. Rokicki D, Zdanowski R, Lewicki S, et al. Inhibition of proliferation, migration and invasiveness of endothelial murine cells culture induced by resveratrol. Centr Eur J Immunol 2014; 39: 449-54.

25. Kedar E, Palgi O, Golod G, Babai I, Barenholz Y. Delivery of cytokines by liposomes. III. Liposome-encapsulated GM-CSF and TNF-alpha show improved pharmacokinetics and biological activity and reduced toxicity in mice. J Immunother 1997; 20: 180-93.

26. Kiafar F, Siahi Shadbad MR, Valizadeh H. Filgrastim (G-CSF) loaded liposomes: mathematical modeling and optimization of encapsulation efficiency and particle size. Bioimpacts 2016; 6: 195-201.

27. Ó`Fágáin C, Colliton K. Storage and lyophilization of pure proteins. Methods Mol Biol 2017; 1485: 159-90.

28. Bergers JJ, ten Hagen TL, van Etten EW, Bakker-Woudenberg IA. Liposomes as delivery systems in the prevention and treatment of infectious diseases. Pharm World Sci 1995; 17: 1-11.

29. Sercombe L, Veerati T, Moheimani F, Wu SY, Sood AK, Hua S. Advances and challenges of liposome assisted drug delivery. Front Pharmacol 2015; 6: 286.

30. Akbarzadeh A, Rezaei-Sadabady R, Davaran S, et al. Liposome: classification, preparation, and applications. Nanoscale Res Lett 2013; 8: 102.

31. Anderson PM, Hanson DC, Hasz DE, Halet MR, Blazar BR, Ochoa AC. Cytokines in liposomes: preliminary studies with IL-1, IL-2, IL-6, GM-CSF and interferon-gamma. Cytokine 1994; 6: 92-101.

32. Mahmud M, Piwoni A, Filipczak N, Janicka M, Gubernator J. Long-circulating curcumin-loaded liposome formulations with high incorporation efficiency, stability and anticancer activity towards pancreatic adenocarcinoma cell lines in vitro. PLoS One 2016; 11: e0167787.

33. Ong SG, Ming LC, Lee KS, Yuen KH. Influence of the encapsulation efficiency and size of liposome on the oral bioavailability of griseofulvin-loaded liposomes. Pharmaceutics 2016; 8: 25.

34. Colletier JP, Chaize B, Winterhalter M, Fournier D. Protein encapsulation in liposomes: efficiency depends on interactions between protein and phospholipid bilayer. BMC Biotechnol 2002; 10: 2:9.

35. Costa AP, Xu X, Burgess DJ. Freeze-anneal-thaw cycling of unilamellar liposomes: effect on encapsulation efficiency. Pharm Res 2014; 31: 97-103.

36. Hernández-Caselles T, Villalaín J, Gómez-Fernández JC. Stability of liposomes on long term storage. J Pharm Pharmacol 1990; 42: 397-400.

37. El-Nesr OH, Yahiya SA, El-Gazayerly ON. Effect of formulation design and freeze-drying on properties of fluconazole multilamellar liposomes. Saudi Pharm J 2010; 18 217-24. 